\title{
Challenges in Observing the Formation of Colloidal, Self-Assembled Monolayers with In Situ Electron Microscopy in Liquid
}

Peter Kunnas ${ }^{1}$, Sebastian Rzadkiewicz ${ }^{2}$, Mohammad-Amin Moradi $^{2}$, Joseph Patterson ${ }^{2}$, Nico Sommerdijk $^{2}$ and Niels de Jonge ${ }^{1,3}$

1. Leibniz Institute for New Materials, Saarbrücken, Germany.

2. Eindhoven University of Technology, Eindhoven, Netherlands.

${ }^{3 .}$ Department of Physics, Saarland University, Saarbrücken, Germany.

Our goal is to develop liquid-phase electron microscopy (LPEM) for observing colloidal assembly processes in situ. Direct imaging methods could possibly elucidate some of the nanoscale interactions that are not well described by models such as Derjaguin-Landau-Verwey-Overbeek-theory [1]. Here, we have made attempts to study a simple system exhibiting colloidal self-assembly, namely the formation of a colloidal self-assembled monolayer (SAM) of positively charged silica nanoparticles $(\mathrm{SiONP})$ on the silicon nitride $(\mathrm{SiN})$ membrane used as liquid cell window. Visualisation could bring new insight to the colloidal organization and create a deeper understanding for observing colloidal interactions with the LPEM in general. The special interest now would be how the process develops from random surface adsorption [2] towards denser monolayer.

A first question is whether SAM formation takes place on the $\mathrm{SiN}$ window and this was tested in bulk liquid. In $\mathrm{pH} 2$, positively charged silica nanoparticles (SiONPs) form a SAM on a plasmatreated $\mathrm{SiN}$ window in bulk liquid. However, the resulting coverage is much lower in $\mathrm{pH}$ 7, (Figure 1b). SAM formation is presumably favourable at low $\mathrm{pH}$ due to the electrostatic interaction between the protonated amino groups of SiONPs and the oxygen-rich window surface [3].

Secondly, we asked the question if SiONPs are visible with sufficient contrast in LPEM. By using high angle angular dark field scanning transmission electron microscopy (HAADF-STEM), we successfully visualized the silica adsorbed on the window in $300-500 \mathrm{~nm}$ thick liquid layer and validated the contrast with Monte-Carlo simulations [4]. (Figure 1a) This required also the accurate measurement of the liquid thickness using Electron energy-loss spectrometry (EELS) log-ratio technique [5]. To differentiate between low-Z material such as SiONP and an artefact may not be trivial. Validation of the contrast by using Monte-Carlo simulations or numerical methods can help to understand the arising contrast from different components of the experiment. We have also conducted exsitu energy dispersive x-ray (EDX) experiments to check the constituents of our sample.

Being able to see SiONPs in liquid, we have attempted to observe the SAM formation in situ by three different methods shown in Figure 2. a) Directly loading the SiONPs in pH 2. This worked but SAM formation had already completed prior to STEM. b) Directly loading the SiONP suspension in $\mathrm{pH} 7$ and inducing the adsorption process by flowing in buffer with $\mathrm{pH}$ 2. c) Flowing the SiONP suspension in $\mathrm{pH}$ 2. Our observations suggest that there is hardly any surface adsorption taking place in confined space ( $b$ and $c$ ). This could be due to the low number of nanoparticles available in the small 300-500 $\mathrm{nm}$ thin volume of liquid. In conclusion, it is possible to observe SiONP in a ready formed SAM but a method still needs to be developed to start the SAM formation to allow observing the process from the early phase. One idea is to change the LP-EM setup by reducing the required diffusion length from bulk liquid to the viewing area. Alternatively, we could use highcontrast materials in a thicker layer of liquid. [6]

References:

[1] CA Silvera Batista et al, Science 350 (2015), p.176.

[2] M Sadowska et al, Langmuir 30 (2014), p.692. 
[3] KW Nichols, Nanofluidics (2017), p.190.

[4] H Demers et al, Microsc Microanal 16 (2010), p.795.

[5] KL Jungjohann et al, Microsc Microanal 18 (2012), p. 621.

[6] We thank Eduard Arzt for his support through INM and Marie Sklodowska-Curie project MULTIMAT.
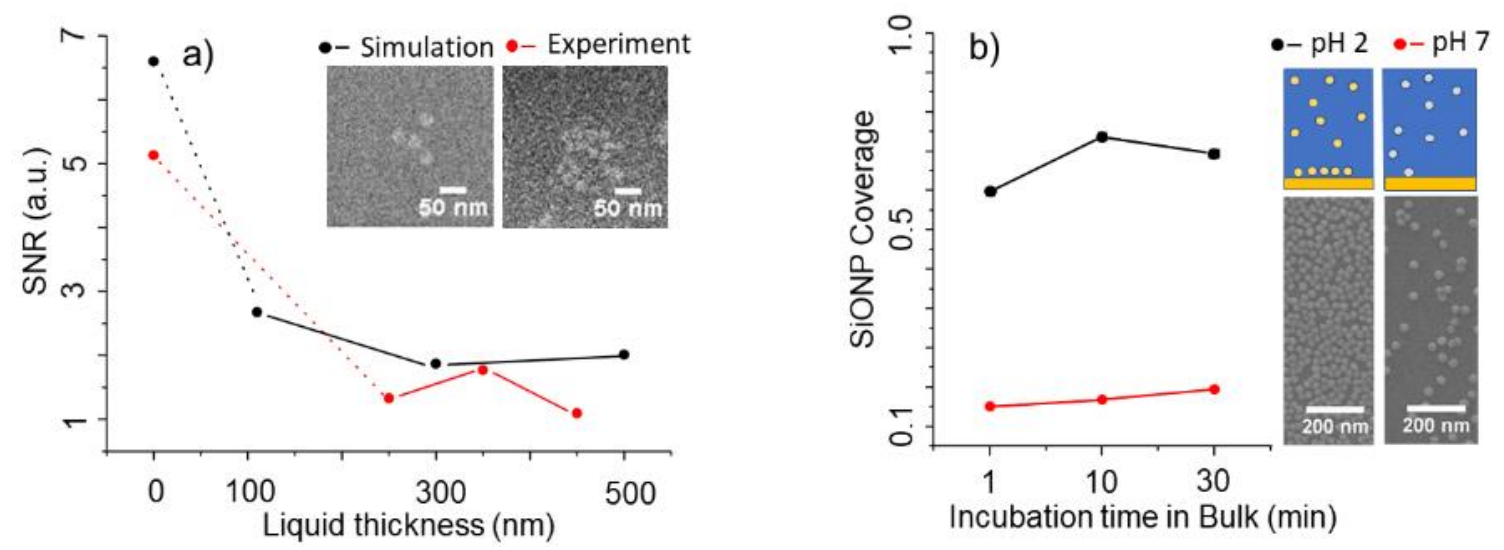

Figure 1. a) Graph shows a comparison between the simulated and experimental HAADF-STEM signal-to-noise ratio (SNR) for SiONPs in liquid. Pixel size $3.1 \mathrm{~nm}$ and electron dose $24 \mathrm{e}^{-} / \mathrm{a}^{2}$. The inset shows image data obtained from simulation and experiments in a liquid thickness of $350 \mathrm{~nm}$. b) The formation of a SAM in bulk liquid takes place quickly and in low $\mathrm{pH} 2$ liquid so that the surface coverage of the SiN membrane is high as seen from the scanning electron microscopy image acquired of a dried sample The surface coverage is much lower at $\mathrm{pH}$ 7. Presumably, the $\mathrm{SiN}$ membrane and amino terminated SiONP carry opposite charges in low $\mathrm{pH}$ favouring the rapid adsorption process.

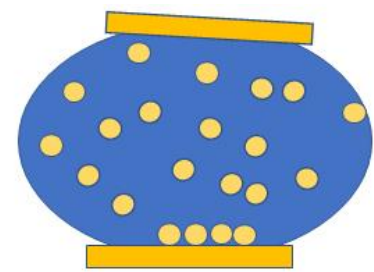

a) Direct loading in $\mathrm{pH} 2$

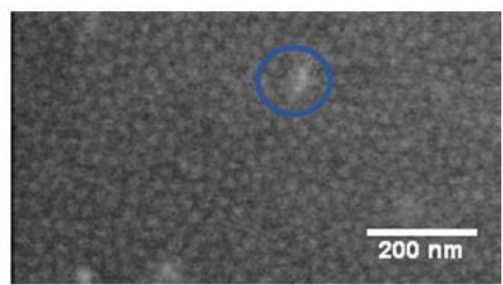

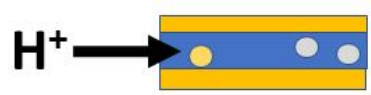

b) Direct loading in $\mathrm{pH} 7$ flow in buffer $\mathrm{pH} 2$

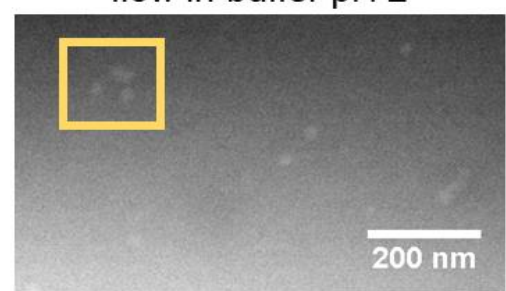

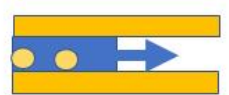

c) Flow in the liquid in $\mathrm{pH} 2$

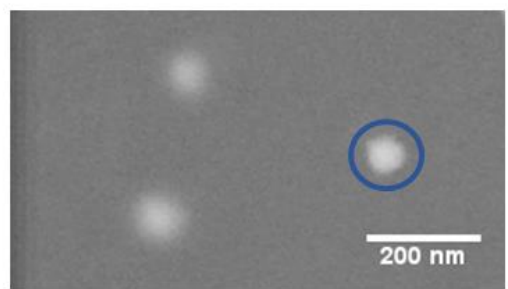

Figure 2. Three different loading methods were tested to observe the SAM formation in situ. a) Direct loading in $\mathrm{pH}$ 2. b) Direct loading in $\mathrm{pH} 7$ and subsequent lowering of the $\mathrm{pH}$ by flowing in acidic buffer c) Directly flowing in the SiONP in $\mathrm{pH}$ 2. Only direct loading in $\mathrm{pH} 2$ resulted with SAM while individual particles are observed also in b) (yellow square). In all of the cases, bright artefacts were observed due to an unknown reason (blue circles). 